Rev. Biol. Trop., 47(1-2): 101-108, 1999

www.ucr.ac.cr www.ots.ac.cr www.ots.duke.edu

\title{
Los quetognatos (Chaetognatha) del banco de Campeche en abril-mayo de 1986
}

\author{
Silvia Mille-Pagaza y Jorge Carrillo-Laguna
}

Lab. de Ecología, Escuela Nacional de Ciencias Biológicas I.P.N. México, D.F., México. C.P. 11 340. México, D.F. Fax (5) 39635 03.Correo electrónico: Jcarrill@alquimia.encb.ipn.mx.

Recibido 12-VIII-1997. Corregido 20-XI-1998. Aceptado 7-XII-1998.

\begin{abstract}
The chaetognaths of the Campeche Bank were studied in April-May, 1986. Organisms were collected at 24 sampling stations with bongo trawls. The taxonomical identity of the specimens collected was determined with basis on McLelland's key and descriptions. We calculated density (expressed as a number of organisms per $100 \mathrm{~m} 3$ ), frequency of occurrence and Importance Value (as the sum of relative density and frequency) for each species and species richness for each sampling station. A dendrogram of sampling stations was constructed using the Morisita`s similarity index and the unweighted pair-group method that uses arithmetic means. Twelve species were recorded. The highest species richness was found at sampling stations located near the continental slope, whereas only one or two species were recorded at the Campeche Sound. Flaccisagitta enflata was the most abundant and widely distributed species in the study area; Sagitta tenuis was the second most abundant species and it ocurred in $79 \%$ of the sampling stations. Krohnitta pacifica and Flaccisagitta lyra were the least frequent species. The highest density of quetognaths $\left(>5000\right.$ organisms $\left./ 100 \mathrm{~m}^{3}\right)$ was recorded at some stations located at the Campeche Sound; values from 1000 to 5000 orgs./100 $\mathrm{m}^{3}$ were recorded in the Western part of the Yucatan Peninsula. Three species groups could be recognised with basis on the species` Importance Values: The first group (IV:> 75) includes F. enflata, S. tenuis, Ferosagitta hispida, K. subtilis and Serratosagitta serratodentata. The second group (IV:>36) comprises four species and the third group (IV:<15) includes the least frequent and abundant species Mesosagitta decipiens, K. pacifica and Flaccisagita lyra. From the dendrogram constructed from the Morisita`s similarity index, five groups of sampling stations were distinguished. The first group included two stations located off the Northern part of the continental slope, where M. minima was the dominant species and the Simpson`s dominance index reached its lowest value (0.215). Most of the species recorded in these stations have an oceanic distribution. The second group comprised seven stations located near the upwelling zone, where F. enflata coexists with five to nine other species and the Simpson`s dominance index had an intermediate value $(0.243)$. The species recorded in these stations have an essentially oceanic distribution and occur northwards from Cabo Catoche. The third group included 10 stations, most of which were located westward from the Campeche Sound and another one in the Yucatan Channel. In these stations F. enflata was the dominant species and the Simpson`s index reached its highest value (0.683). The fourth group included one station located in the upwelling zone, were the dominance index has its lowest value and S. serratodentata was the dominant species. The fifth group included coastal stations where $F$. enflata was the dominant species and the Simpson`s index reached high values. The most important characteristics of the study area during this period were: 1. Two distinct zones. The Campeche Sound in the Western part of the area and the Northern coastal stations, where $F$. enflata was the dominant species and the Simpson`s dominance index reached its highest values, and the oceanic Northern zone off the Yucatan Peninsula where $F$. enflata had low abundance and there was a higher abundance and diversity of other chaetognath species. 2. F. enflata, S. tenuis, F. hispida and K. subtilis were the dominant chaetognath species.
\end{abstract}

Key words: Chaetognatha, species richness, Campeche Bank, zooplankton, similarity, abundance. 
Los quetognatos constituyen un grupo ampliamente estudiado en la región norte del golfo de México (Pierce 1951, 1954, 1962, Owre 1960, McLelland 1984, 1989), cuyo interés se sostiene en la importancia de las especies del grupo como indicadoras de ciertas condiciones ambientales (Alvariño 1965, 1969, Michel y Foyo 1976), y por ser depredadoras de larvas de peces o de otras especies zoopláncticas. Sin embargo, en el resto del golfo, sobre todo en el área correspondiente a la Zona Económica Exclusiva de México, los estudios que sobre el grupo se tienen (VegaRodríguez 1965, Rivero 1975, Mille et al. 1997) son muy escasos.

La plataforma yucateca, conocida también como banco de Campeche, localizada al sureste del golfo es particularmente interesante por la influencia que sobre ella ejerce la corriente de Yucatán proveniente del mar Caribe que, al chocar con el talud de dicha plataforma, provoca el afloramiento de aguas profundas creando una surgencia que se expresa frente a cabo Catoche y frente a puerto Progreso, al norte de la península de Yucatán (Suárez 1990, Merino-Ibarra 1994).

El agua que aflora en la porción norteña del banco es rica en nutrientes (Belousov et al. 1966, Bogdanov et al. 1968), lo que determina una amplia proliferación de especies de herbívoros que a su vez son utilizados como alimento por grupos de carnívoros como los quetognatos y sifonóforos (Gasca y Suárez 1991 a, b). En cambio, la parte oeste de la plataforma, conocida como sonda de Campeche, se caracteriza por ser una zona relativamente somera e influenciada por aguas superficiales con elementos de las surgencias y aguas profundas procedentes del mar Caribe (Nowlin 1971).

Las condiciones fisiográficas e hidrológicas que prevalecen en estas dos zonas de la plataforma yucateca hacen pensar que los ambientes son distintos y por ende también la composición local del grupo Chaetognatha. Se pretenden definir estas diferencias mediante el análisis de la composición específica, las abundancias y la distribución de los quetognatos en las dos zonas durante abril-mayo de 1986.

\section{MATERIALES Y MÉTODOS}

Las muestras de zooplancton fueron recolectadas mediante arrastres oblicuos con red bongo de $300 \mu \mathrm{m}$ de malla a bordo del buque oceanográfico "Justo Sierra" (campaña JS 8601) en abril-mayo de 1986, por personal del Instituto Nacional de la Pesca. Los quetognatos se obtuvieron de 24 muestras correspondientes a estaciones localizadas al norte y suroeste de la plataforma yucateca, recolectadas desde los 13.7 hasta los 211 metros de profundidad estimada (Fig. 1, Cuadro 1).

La identificación del material se realizó con las claves y descripciones de McLelland (1989) siguiendo la clasificación de Bieri (1991). Se determinó la riqueza específica por estación, la frecuencia de aparición y se estimó la densidad por especie (organismos $/ 100 \mathrm{~m}^{3}$ ). Se calculó el Valor de Importancia (Krebs 1985) sumando las abundancias y frecuencias relativas de cada especie y se utilizaron la similitud de Morisita y el método de ligamiento de promedios no ponderados para agrupar las

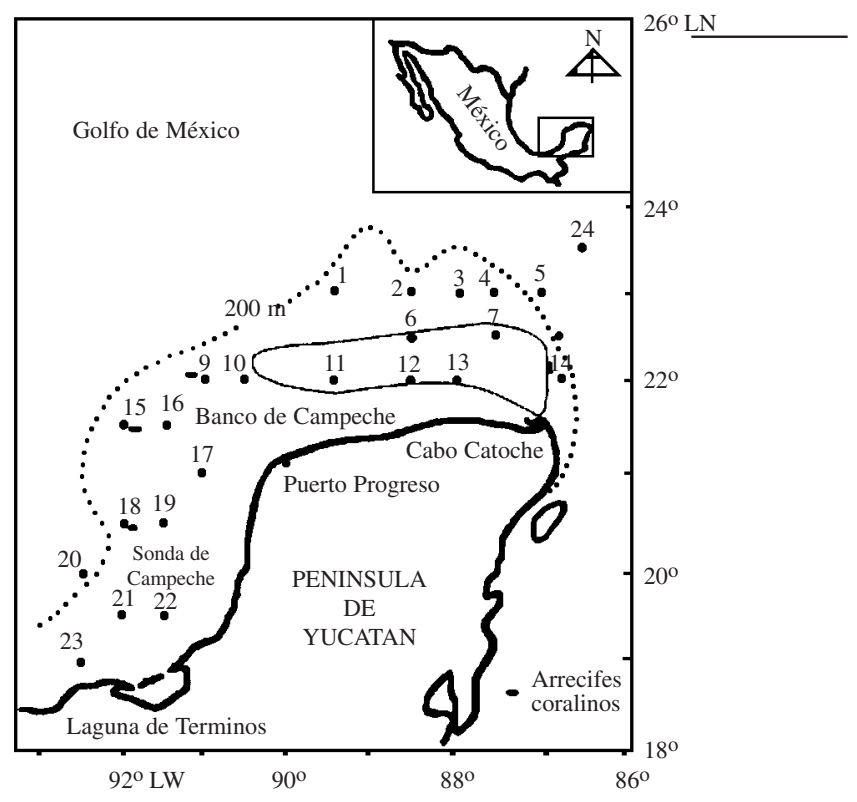

Fig. 1. Area de estudio. Posición de estaciones y ubicación de la surgencia. 


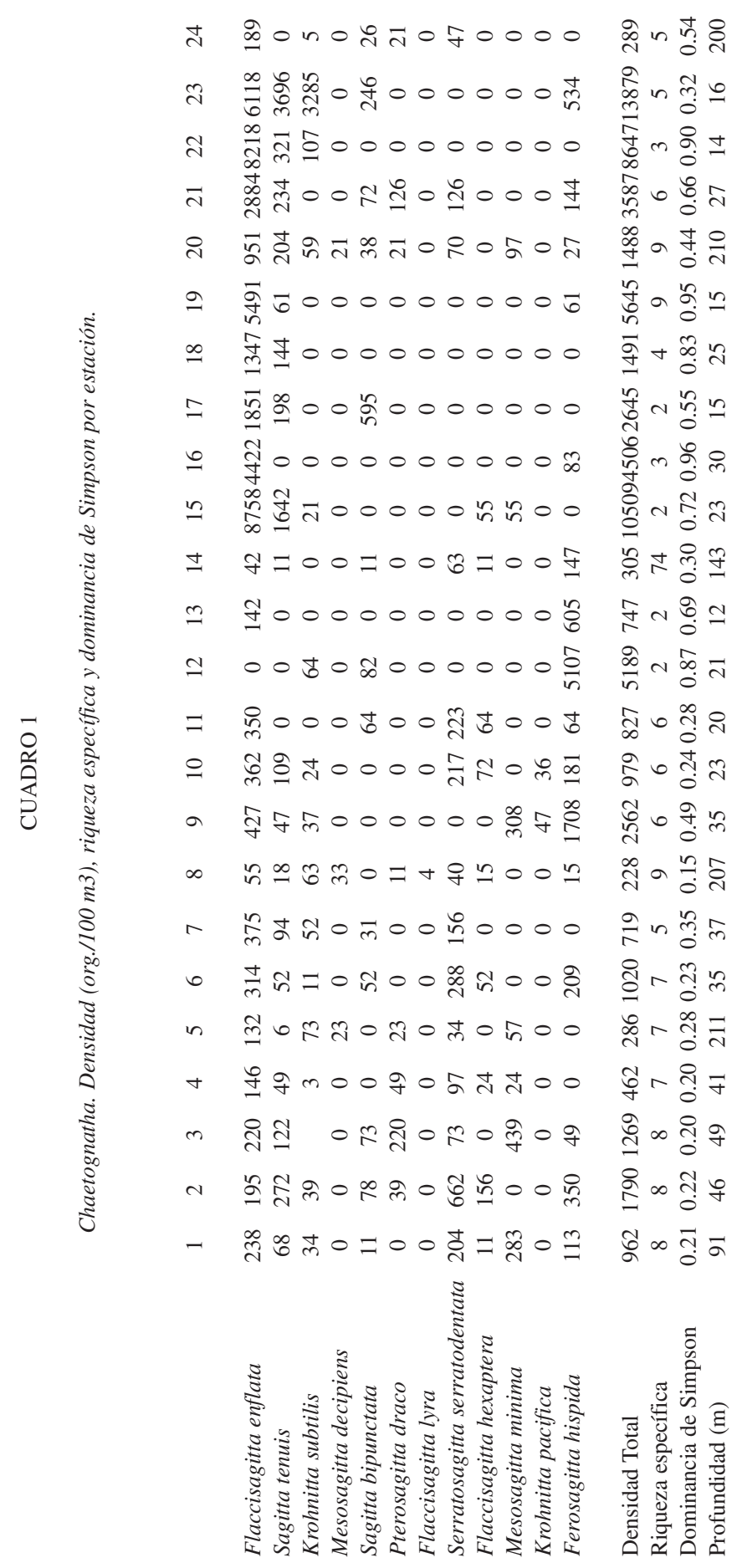


estaciones de acuerdo a su semejanzas en composición y abundancia. Como una medida de la diversidad se calculó la dominancia de Simpson para cada estación y un promedio para los grupos, donde fue posible (Ludwig y Reynolds 1988).

\section{RESULTADOS}

Se recolectaron doce especies de quetognatos cuya lista se presenta en el cuadro 2 , ordenada de acuerdo con su Valor de Importancia (VI). El mayor número de especies, de ocho a nueve, lo presentaron las estaciones 1 , $2,3,8$ y 20 , todas cerca de la orilla de la plataforma (Fig. 1). En las estaciones localizadas en la zona norte del banco de Campeche se encontraron entre cinco y siete especies mientras que, en dos costeras de esta región y en la mayor parte de la sonda de Campeche, se registraron únicamente entre dos y cuatro especies.

Las especies encontradas en el banco de Campeche durante esta época tienen distribución tropical-templada y son epiplanctónicas (Cuadro 2) con excepción de Sagitta tenuis y Ferosagitta hispida que son del Atlántico tropical y neríticas
(Owre 1960, McLelland 1984, McLelland y Heard 1991).

En el área de estudio la especie con más amplia distribución fue Flaccisagitta enflata que apareció en el 99\% de las estaciones (Cuadro 1), con excepción de la 12 situada al norte de la península de Yucatán. En segundo lugar, se registró a $S$. tenuis en el $79 \%$ de las estaciones. Las menos frecuentes fueron Krohnitta pacifica y Flaccisagitta lyra, presentes en dos y una estación, respectivamente.

En las estaciones 15, 19, 22 y 23 localizadas en la Sonda de Campeche la densidad de quetognatos fue superior a los 5000 org. por $100 \mathrm{~m}^{3}$ (Cuadro 1); las estaciones del oeste de la península mostraron valores entre 1000 y 5 000 quetognatos por $100 \mathrm{~m}^{3}$. Se encontraron densidades similares en la zona de surgencia al centro de la región norteña.

Los cálculos del Valor de Importancia indican que las especies de quetognatos presentes en ese período se separan en tres niveles (Cuadro 2), en el primero, con valor superior a 75 , en orden decreciente quedaron $F$. enflata, S. tenuis, F. hispida, K. subtilis y Serratosagitta serratodentata. Un segundo nivel lo forman cuatro especies con valor superior a $36 \mathrm{y}$ en el

CUADRO 2

Elenco taxonómico, Valor de Importancia (VI) y distribución mundial de los quetognatos encontrados en el Banco de Campeche, México en abril-mayo de 1986

Especies

Flaccisagitta enflata (Grassi, 1881)

Sagitta tenuis Conant, 1896

Ferosagitta hispida (Conant, 1895)

Krohnitta subtilis (Grassi, 1881)

Serratosagitta serratodentata (Krohn, 1853)

Sagitta bipunctata Quoy y Gaimard, 1827

Flaccisagitta hexaptera (d'Orbigny, 1843)

Mesosagitta minima (Grassi, 1881)

Pterosagitta draco (Krohn, 1853)

Mesosagitta decipiens (Fowler, 1905)

Krohnitta pacifica (Aida, 1897)

Flaccisagitta lyra (Krohn, 1853)
VI $(\%)$

118.9

98.2

82.7

82.2

72.3

67.2

46.5

36.2

36.2

15.5

10.3

5.17
Distribución mundial *. O-epip.,templada-tropical. Nerítica, atlántico-trópico-ecuatorial Costera.-epip., atlántico-trópico-ecuatorial. O-epip., templada-tropical.

O-epip., atlántico-templada-tropical.

O-epip., tropical-templada.

O-meso, tropical-templada.

O-epip., tropical templada.

O-epip., tropical-templada.

O-meso.-batipelágica, tropical-templada.

O-epip., tropico-ecuatorial.

O-epip., tropical-templada.

(O) Oceánica , (epip.) epiplanctónica, (meso) mesoplanctónica. 
tercer nivel quedan únicamente las tres especies menos frecuentes y abundantes del muestreo.

Con el índice de Morisita entre estaciones se separan cinco grupos con un nivel de similitud superior al $75 \%$ (Fig. 2)

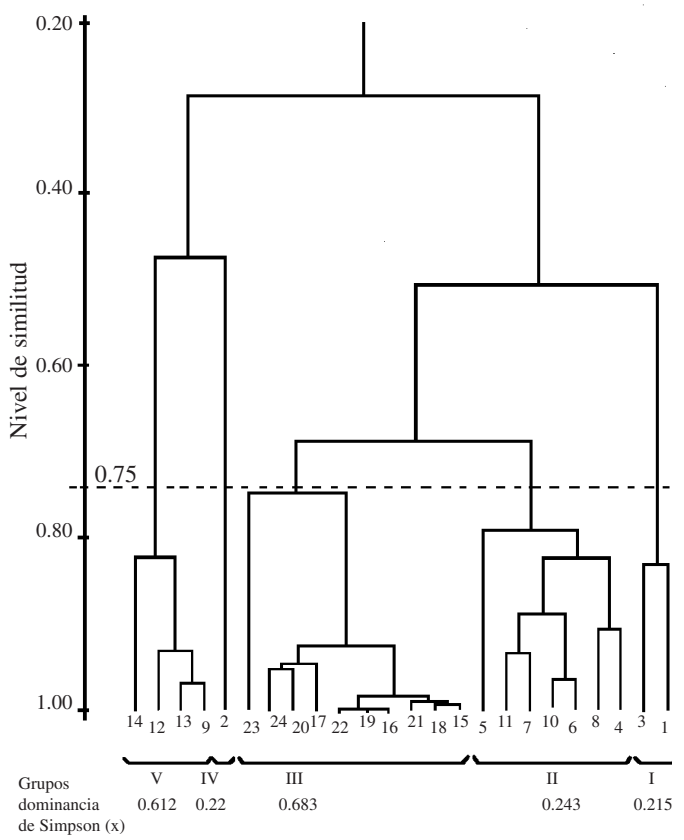

Fig. 2. Agrupación de estaciones mediante la similitud de Morisita y dominancia promedio de Simpson.

\section{DISCUSIÓN}

La riqueza específica encontrada en este período muestra una pequeña diferencia con lo reportado por Rivero (1975) quien recolectó 10 especies para la región de la sonda de Campeche, sin embargo, en nuestro trabajo no se encontró a Sagitta friderici que esta autora reportó como una de las más conspicuas de la sonda, junto con otras especies menos abundantes, que tampoco aparecieron en este crucero; estas diferencias se podrían atribuir a un período diferente de muestreo o como lo marca Tokioka (1974) al hecho de que son formas ecológicas de la misma especie, siendo $S$. tenuis la forma típica.

Las condiciones tan particulares de la plataforma de Yucatán, en cuanto a ser una zona relativamente somera con poca influencia de aguas continentales (de la Lanza-Espino 1991), podrían ser determinantes en la baja diversidad local de quetognatos, notoriamente diferente de la reportada para el Caribe, donde Michel y Foyo (1976) reportaron 22 especies, de las cuales las más abundantes fueron, en su mayoría, las mismas que se encontraron en este período para la plataforma yucateca, con excepción de $S$. tenuis que no apareció en el estudio realizado para el Caribe, posiblemente por ser una especie estrictamente nerítica (Alvariño 1965, Boltovskoy 1981).

En el estudio de Owre (1960), en la corriente de Florida, se encontraron 16 especies, entre las que están ocho de las que aparecen en este estudio; tampoco en la corriente de Florida aparece $S$. tenuis, sin embargo, hacia el norte y noreste del golfo esta especie ha sido reportada en los estudios realizados por Pierce (1951, 1962), Suárez-Caabro (1955), Alvariño (1969), McLelland (1978, 1984) y en México en una zona muy costera situada frente a Veracruz (Vega-Rodríguez 1965) lo que confirma su afinidad nerítica (Alvariño 1965, Michel y Foyo 1976).

Las elevadas densidades obtenidas en la mayor parte de las estaciones de la sonda y al norte de la plataforma se atribuyen fundamentalmente a la alta concentración de F. enflata, que por su carácter eurioico ha sido catalogada como abundante en aguas costeras (Owre 1960); divergen de este patrón dos estaciones donde domina $F$. hispida la cual tiene preferencia por las aguas costeras (Pierce 1962, Pierce y Wass 1962, McLelland y Heard 1991) y es característica de aguas del golfo de México (Pierce 1951, Owre 1960).

De las cinco especies con VI superior a 75 (Cuadro 2) S. serratodentata es la más común en la Corriente de Florida (Owre 1960), pero dada su afinidad oceánica, en el banco de Campeche se encontró restringida al norte de la plataforma donde la influencia de la corriente de 
Yucatán es más marcada. Estas cinco especies se han catalogado como tropicales o templadas características del Atlántico central; varias de ellas son indicadoras de aguas costeras o de talud (Alvariño 1965, Michel y Foyo 1976, McLelland 1989), lo que justifica su amplia distribución en esta plataforma. S. tenuis abarcó casi toda el área de estudio aunque con valores bajos, posiblemente debido a la falta de aportes fluviales importantes en el área (Alvariño 1968).

Las especies con Valor de Importancia bajo son oceánicas o indicadoras de surgencias como Mesosagitta decipiens, $K$. pacifica y $F$. lyra (Alvariño 1968) y se encontraron restringidas a las estaciones cercanas al canal de entrada de la corriente de Yucatán o a la zona suroeste de la sonda donde se ha detectado una pequeña surgencia y por tanto mezcla de aguas.

Del conjunto de especies con Valor de Importancia medio M. minima, característica de aguas costeras o de mezcla y que Alvariño (1968) reporta como escasa para el Caribe y Golfo de México y abundante en la Corriente del Golfo no es registrada por Suárez-Caabro (1955) y Suárez-Caabro y Madruga (1960) en Cuba y Honduras y tampoco fue encontrada por Rivero (1975) en la sonda de Campeche; sin embargo, Michel y Foyo (1976) la observaron como la segunda en abundancia en el Caribe, posiblemente como indicadora de surgencias; en este trabajo también se le encontró asociada con la zona de surgencia. Además integraron este grupo Pterosagitta draco, Flaccisagitta hexaptera y Sagitta bipunctata, todas oceánicas. Las dos primeras se distribuyeron principalmente al norte de la península, en la zona de mayor influencia de la corriente de Yucatán. La presencia de $P$. draco en la zona del talúd frente a la laguna de Términos podría deberse a la mezcla de aguas costeras y oceánicas, condiciones en las que otros autores la han reportado (Pierce 1962, Pierce y Wass 1962, McLelland 1984).

De acuerdo con Alvariño (1968) la presencia de $P$. draco se relaciona inversamente con $S$. bipunctata; en el área de estudio ambas coincidieron en varias estaciones con densidades inversas. Esta última especie fué catalogada como relativamente rara para el Caribe (Michel y Foyo 1976) y en este crucero se encontró en el 50\% de las estaciones aunque con densidades bajas.

La distribución de las especies de quetognatos encontradas y de sus abundancias nos indican que en el banco de Campeche existen dos zonas diferentes: la sonda de Campeche y la región norte y noreste de la península. Lo anterior se confirma con los cinco grupos formados mediante la aplicación del índice de similitud de Morisita entre estaciones (Fig. 2); en el primer grupo, con dos estaciones localizadas al norte de la plataforma, domina M. minima, sobre otras siete especies lo que se refleja en el valor promedio mas bajo del índice de Simpson (0.215). El segundo y tercer lugar en abundancia dentro del grupo lo ocuparon $F$. enflata y P. draco. La mayor parte de las especies presentes fueron oceánicas (Fig. 3).

El segundo grupo tiene siete estaciones cercanas a la surgencia donde $F$. enflata, coexiste con cinco a nueve especies, lo que se refleja en un valor promedio de dominancia bajo aunque algo mayor al anterior (0.243). El ensamblaje que se forma es esencialmente oceánico y se presenta en el límite externo de la plataforma al norte de cabo Catoche (Fig. 3). En la estación ocho que integra este grupo, $M$. decipiens y $F$. lyra, dos de las especies con menor Valor de Importancia, presentan sus máximas densidades.

Otro grupo lo integran 10 estaciones (Fig. 2); la mayoría al oeste en la sonda de Campeche y una en el canal de Yucatán (Fig. 3). Domina $F$. enflata de manera absoluta y en segundo lugar S. tenuis, que se traduce en el valor promedio de dominancia más grande registrado (0.683). En la mayoría de las estaciones de este grupo se obtuvieron sólo otras dos o tres especies, lo cual sugiere una exclusión competitiva por parte de $F$. enflata en el área o la presencia de condiciones ambientales más adecuadas para esta especie. K. subtilis y S. bipunctata así como $S$. tenuis tienen en este grupo sus mayores densidades; aunque $F$. enflata las supera aproximadamente por el doble. 


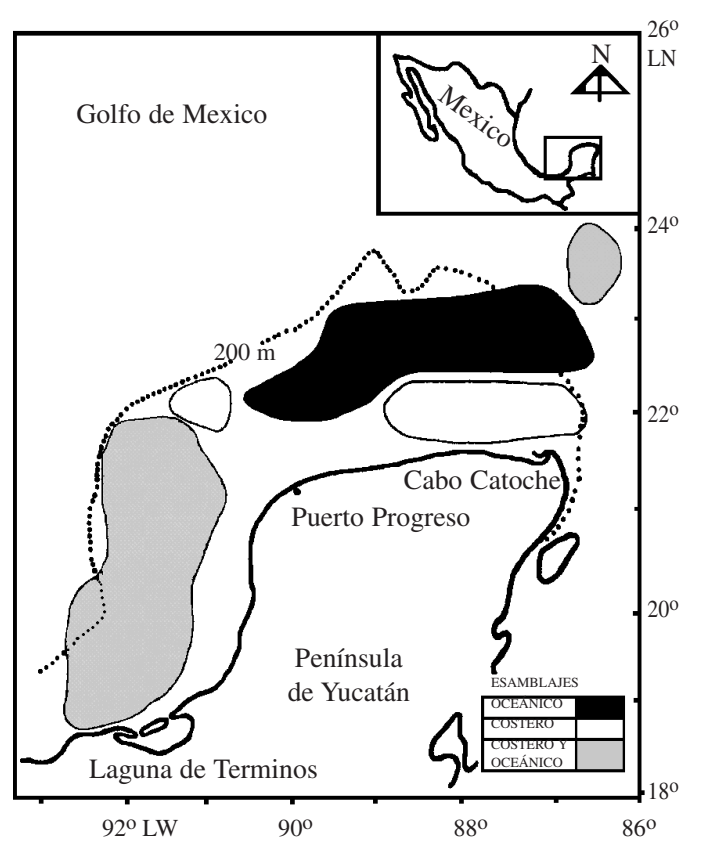

Fig. 3. Ensamblaje de especies de quetognatos del banco de Campeche en abril mayo de 1986.

El cuarto grupo lo constituye una estación de la zona de influencia de la surgencia (Fig. 3), el valor de dominancia promedio fue el segundo más bajo de todos (0.22), destaca la especie oceánica $S$. serratodentata. En esta misma estación $F$. hexaptera tuvo su densidad más alta.

El quinto grupo donde la dominancia promedio fue la segunda más alta (0.612) se define como costero (Fig. 3) con un claro predominio de $F$. hispida sobre el resto de las especies e incluso, en las estaciones 12 y 13 donde esta especie mostró sus máximas densidades, F. enflata presentó la mínima y en una de ellas no se encontró; ésto también nos habla de una posible exclusión competitiva aún mas fuerte que la de $F$. enflata mencionada antes o, de requerimentos diferentes para estas especies. En la estación nueve que forma parte de este grupo, una de las especies mas escasas del área, $K$. pacifica, tuvo su densidad máxima.

Los procedimentos utilizados muestran una notable concordancia en cuanto a la definición de los principales ensamblajes en la plataforma, por lo que podemos decir que el área de estudio en este período se caracteriza por:

1.- Dos zonas: a) una que comprende algunas estaciones costeras al norte del Banco y las del oeste de la península de Yucatán (sonda de Campeche) donde $F$. enflata domina francamente, lo que se refleja en los valores promedio más altos de la dominancia de Simpson y b) la oceánica al norte de la península donde las variaciones en las condiciones hidrológicas del área favorecen la diversidad y mayor abundancia de otras especies de quetognatos y determinan la disminución de la abundancia de $F$. enflata.

2.- Cuatro especies dominantes: F. enflata, S. tenuis, F. hispida y K. subtilis.

\section{AGRADECIMIENTOS}

Agradecemos profundamente a Salvador Sánchez Colón por sus atinadas observaciones en la elaboración del manuscrito y a todos aquellos revisores anónimos por sus invaluables comentarios

\section{RESUMEN}

Al analizar la distribución, frecuencia, abundancia y Valor de Importancia de 12 especies de quetognatos encontradas en 24 estaciones de muestreo durante abril-mayo de 1986 en el banco de Campeche, se observa que la mayor riqueza específica ocurrió en estaciones oceánicas cerca del talud, en tanto que los sitios con mayor densidad de quetognatos (más de 5000 org./ $100 \mathrm{~m}^{3}$ ) se ubicaron en la parte oeste del área de estudio. Las especies que formaron el elenco taxonómico son principalmente oceánicas, de aguas tropicales y subtropicales, de éstas las que obtuvieron los mayores Valores de Importancia fueron: Flaccisagitta enflata, Sagitta tenuis, Ferosagitta hispida, Krohnitta subtilis y Serratosagitta serratodentata. La similitud de Morisita entre estaciones y la dominancia de Simpson permiten definir dos regiones: una de ellas formada por las estaciones norteñas costeras y las de la sonda de Campeche donde domina $F$. enflata y los valores de dominancia de Simpson fueron altos y la otra que comprende las estaciones oceánicas norteñas del Banco de Campeche donde no hubo una especie claramente dominante y por tanto los valores del índice de Simpson fueron bajos. 


\section{REFERENCIAS}

Alvariño, A. 1965. Chaetognaths. Ann. Rev. Oceanogr. Mar. Biol. 3:115-194.

Alvariño, A. 1968. Los quetognatos, sifonóforos y medusas en la región del Atlántico ecuatorial bajo la influencia del Amazonas. An. Inst. Biol. Univ. Nal. Autón. México. 39, Ser. Cienc. del Mar y Limnol. (1): 41-76.

Alvariño, A. 1969. Los quetognatos del Atlántico. Distribución y notas esenciales de sistemática. Trab. Inst. Esp. Oceanogr. 37. 290 p.

Belousov, I.M., Y.A. Ivanov, S.A. Pasternak, T.S. Rass \& V.V. Rossov.1966. Oceanographic Research by the Soviet-Cuban Marine Expedition. Okeanology 6: 312-320.

Bogdanov, D. V., A. Sokolov \& N.S. Krohmov.1968. Regions of high biological and commercial productivity in the Gulf of Mexico and Caribbean Sea. Okeanology 8: 371-381.

Boltovskoy, D. 1981. Chaetognatha, p. 759-791 In: D. Boltovskoy (ed). Atlas del Zooplancton del Atlántico sudoccidental. , Publ. Inst. Nac. Invest. y Des. Pesc. (INIDEP), Mar del Plata, Argentina.

de la Lanza-Espino, G. (Comp.). 1991. Oceanografía de mares mexicanos. AGT, México, D.F. 569 p.

Gasca, R. \& E. Suárez. 1991a. Siphonophores and upwelling in the Mexican Caribbean Sea. Plankton Newsl. 14: 5-8.

Gasca, R. \& E. Suárez. 1991b. Siphonophores of upwelling areas of the Campeche Bank and Mexican Caribbean Sea. Hidrobiologia 216/217: 497-502.

Krebs, C.J. 1985. Ecología. Estudio de la distribución y la abundancia. Harla, México, D.F. 753 p.

Ludwig, J.A. \& J.F. Reynolds. 1988. Statistical Ecology. Wiley, Nueva York. 337 p.

McLelland, J.A. 1978. The summer distribution of Chaetognatha in the northeastern Gulf of Mexico. M.S. Thesis, University of Southern Mississippi, Hattiesburg, Mississippi $173 \mathrm{p}$.

McLelland, J.A.. 1984. Observations on chaetognath distributions in the northeastern Gulf of Mexico during the summer of 1974. Northeast Gulf Sci. 7: 49-59.

McLelland, J.A.. 1989. An Illustrated key to the Chaetognatha of the Northern Gulf of Mexico with notes on their distribution. Gulf Res. Rep. 8: 145-172.
McLelland, J.A. \& R.W. Heard. 1991. Notes on some chaetognaths from Pine Cay, Turks and Caicos Islands (British West Indies). Gulf Res. Rep. 8: 227-235.

Merino-Ibarra, M. 1994. Afloramientos en la Plataforma de Yucatán, estructura y fertilización (síntesis). Oceanología. Unidad de Educación en Ciencias y Tecnología del Mar. S.E.P. México 1: 139-144.

Michel, H.B. \& M. Foyo. 1976. Caribbean zooplankton. Part I - Siphonophora, Heteropoda, Copepoda, Euphausiacea, Chaetognatha and Salpidae. Office of Nav. Res. Dept. of Navy, U.S. Govt. Printing Office, Washington, D.C. 549 p.

Mille-Pagaza, S., Reyes-Martínez \& M. E. Sánchez-Salazar. 1997. Distribution and abundance of Chaetognatha on the Yucatan Shelf during May, 1986. Gulf Res. Rep. 9: 263-275.

Nowlin, W.D. 1971. Water masses and general circulation of the Gulf of Mexico. Texas A \& M Univ. Contribution 452: 28-33.

Owre, H.B. 1960. Plankton of the Florida Current. Part VI. The Chaetognatha. Bull. Mar. Sci. 10: 255-322.

Pierce, E.L. 1951.The Chaetognatha of the west coast of Florida. Biol. Bull. (Woods Hole) 100: 206-228.

Pierce, E.L. 1954. Notes on the chaetognaths of the Gulf of Mexico. U.S. Fish Wildl. Serv. Fish. Bull. 55: 227-329.

Pierce, E.L. 1962. Chaetognatha from the Texas coast. Publ. Inst. Mar. Sci. Univ. Tex. 8: 147-152.

Pierce, E. L. \& M.L. Wass. 1962. Chaetognatha from the Florida Current and coastal water of the southeastern Atlantic states. Bull. Mar. Sci. 12: 403-431.

Rivero, B.C. 1975. Distribución de los quetognatos en la Bahía de Campeche. Mem. I Simp. Lat. Ocean. Biol. (México): 306-324.

Suárez-Caabro, J.A. 1955. Quetognatos de los mares Cubanos. Mem. Soc. Cubana Hist. Nat. 22: 125-180.

Suárez-Caabro, J.A. \& J.E. Madruga. 1960. The Chaetognatha of the northeastern coast of Honduras, Central America. Bull. Mar. Sci. 10: 421-429.

Suárez, M. E.1990. Planktonic Copepods: A note on their relation with upwelling areas in the Campeche Bank and the Mexican Caribbean sea. Inv. Mar. CICIMAR 5: 87-92.

Vega-Rodríguez, F. 1965. Distibución de Chaetognatha en Veracruz, Ver. An. Inst. Biol., Univ. Nac. Autón. Méx. Ser. Zool.. 36: 229-247. 